\title{
PSICOLOGIA DO ESPORTE NO BRASIL: CONCEITUAÇÃO E O ES- TADO DA ARTE
}

\author{
Ivan Wallan Tertuliano \\ Centro Universitário Adventista de São Paulo, São Paulo, São Paulo, Brasil.
}

\section{Afonso Antonio Machado}

Universidade Estadual Paulista, Rio Claro, São Paulo, Brasil.

\begin{abstract}
Resumo
O objetivo do presente estudo foi conceituar e delimitar áreas de atuação na Psicologia do Esporte no Brasil e apresentar a evolução da área até o momento atual. Para isso, este estudo visou a refletir sobre a temática, pautando-se na revisão de estudos clássicos e contemporâneos, nacionais e internacionais. Nessa perspectiva, optou-se por apresentar estudos que fazem parte do estado da arte da Psicologia do Esporte e que se encontrassem publicados entre 1980 e 2017. Assim, pode-se concluir que a Psicologia do Esporte é uma área interdisciplinar que surgiu em função do diálogo de diferentes áreas, como a Psicologia e a Educação Física. Além disso, cabe ressaltar que é uma área em que o Profissional de Educação Física e o Psicólogo podem atuar em conjunto e com profissionais de outras áreas.
\end{abstract}

Palavras-chave: Psicologia do Esporte. Educação Física. Psicologia. Estado da arte.

\section{PSYCHOLOGY OF SPORT IN BRAZIL: CONCEPTUALIZATION AND THE STATE OF THE ART}

\begin{abstract}
The objective of the present study was to conceptualize and delimit areas of practice in Sport Psychology in Brazil and present the evolution of the area up to the present moment. For this, this study aimed to weave on the theme, based on the review of classic and contemporary studies, national and international. From this perspective, it was decided to present studies that are part of the state of the art of Sports Psychology and that were published between 1980 and 2017. Thus, it can be concluded that the Psychology of Sport is an interdisciplinary area, which arose as a function of the conversation of different areas, such as Psychology and Physical Education. In addition, it should be noted that this is an area where the Physical Education Professional and the Psychologist can work together with other areas.
\end{abstract}

Keywords: Psychology of Sport. PE. Psychology. State of art.

\section{PSICOLOGÍA DEL DEPORTE EN BRASIL: CONCEPTUALIZACIÓN Y EL ESTA- DO DEL ARTE}

\section{Resumen}

El objetivo del presente estudio fue conceptuar y delimitar áreas de actuación en la Psicología del Deporte en Brasil y presentar la evolución del área hasta el momento actual. Para ello, este estudio visó tejer sobre la temática, pautándose en la revisión de estudios clásicos y contemporáneos, nacionales e internacionales. En esa perspectiva, se optó por presentar estudios que 
forman parte del estado del arte de la Psicología del Deporte y que se encontraran publicados entre 1980 y 2017. Así, se puede concluir que la Psicología del Deporte es un área interdisciplinaria, la cual surgió en función de la conversación de diferentes áreas, como la Psicología y la Educación Física. Además, cabe resaltar que es un área en que el Profesional de Educación Física y el Psicólogo pueden actuar en conjunto con otras áreas.

Palabras clave: Psicología del Deporte. Educación Física. Psicología. Estado del arte.

\section{Introdução}

No mundo atual, o Esporte é visto como um dos maiores espetáculos, atraindo pessoas e despertando o interesse de diversas áreas científicas, como por exemplo, a sociologia, antropologia, biomecânica e psicologia (TERTULIANO, 2016). Todas essas áreas, e outras mais, constituem as Ciências do Esporte que têm o objetivo de contribuir para a melhora da prática esportiva. Alguns estudiosos defendem que a Psicologia do Esporte pode ser entendida como uma subárea da Psicologia (RUBIO, 2003) ou das Ciências do Esporte (GILL, 1986). Weinberg e Gould (2017) apontam que a Psicologia do Esporte é um estudo científico de pessoas em atividade física e/ou esportiva, bem como a aplicação desse conhecimento. Independente da subárea em que esteja inserida, ela tem como objetivo tanto a produção de conhecimento quanto a atuação profissional (VIEIRA; NASCIMENTO JR; VIEIRA, 2013). Isso pode ser constatado mediante o avanço teórico-prático da Psicologia do Esporte que oferece material para atuação em diferentes ambientes esportivos, agregando conhecimento de diferentes paradigmas e correntes teóricas da Psicologia (VIANA-MEIRELES et al., 2015) e das Ciências do Esporte (SCHIAVON; MACHADO, 2012).

Partindo desse prisma, a Psicologia do Esporte, tal como se apresenta no Brasil, resulta em três áreas básicas: a) Estudos sobre Comportamento Motor (Aprendizagem Motora e Desenvolvimento Motor); b) Especulações teóricas e dados de pesquisa acerca da preparação psicológica do atleta de alto rendimento, aliada a diferentes áreas da psicologia (motivação, personalidade, inteligência etc.); c) Pesquisas e estudos sobre a Psicologia Social da equipe e de esportes (BARRETO, 2003). Mas em qualquer uma dessas áreas (teórico-prático) existe uma adequação dos conhecimentos advindos das pesquisas para a atuação prática. Infelizmente, no cenário nacional, a Psicologia do Esporte somente encontra vazão para estudo, pesquisa e intervenção na Ciência do Esporte e, muito sensivelmente, na Fisioterapia, Psicologia e Medicina (MACHADO, 2014).

Todavia, novos olhares, partindo de profissionais com dupla formação, estão possibilitando que o cenário comece a se delinear de modo que o espectro profissional inicie uma estruturação com iniciativas interdisciplinares, pautadas em avanços endossados pela academia. A América do Norte e a Europa têm leituras diferenciadas sobre a delimitação de áreas de atuação da Psicologia do Esporte. Isso traz, para a academia brasileira, certo desconforto diante "do existente" e da necessidade de postura profissional, o que gera desentendimento com conselhos de classes profissionais e uma grande inatividade diante dos avanços possíveis (MACHADO, 2014; SCHIAVON; MACHADO, 2012; VIEIRA; NASCIMENTO JR; VIEIRA, 2013).

Assim, o presente trabalho tem a seguinte problemática norteadora: qual a conceituação da Psicologia do Esporte, qual sua abrangência e quais são suas áreas de atuação no Brasil? Para atender tal problema, o objetivo do presente estudo é conceituar e delimitar áreas de atuação na Psicologia do Esporte no Brasil. Além de apresentar os principais estudos documentais da área, identificando áreas de atuação, formação e intervenção na Psicologia do Esporte Brasileira. 
Dessa forma, com o intuito de apresentar e propor posturas acadêmico-profissionais, este estudo visa trazer à sociedade brasileira de Psicologia do Esporte um direcionamento pautado na revisão de estudos clássicos e contemporâneos, nacionais e internacionais, para justificar a pesquisa e proporcionar um maior raio de atuação na emergente área aqui apresentada. Assim, este ensaio baseou-se no desenho metodológico de uma pesquisa de natureza qualitativa sobre a perspectiva de análise documental (LAKATOS; MARCONI, 2011), visto que a pesquisa se objetiva na compilação de um material adequado para incrementar propostas de avanços das áreas interdisciplinares aqui implicadas e, em especial, favorecer ao conhecimento das áreas de atuação na Psicologia do Esporte (CAMPBELL; MACHADO, 2013). Nesse desenho metodológico buscou-se referencial disponível por meio de buscadores eletrônicos, como: Google acadêmico, Periódicos Capes, PubMed, Science Research, World Wide Science e Scielo. Como forma de busca, optou-se por descritores que atendessem ao objetivo do estudo. Assim, descritores como: Psicologia do Esporte no Brasil, Estado da Arte em Psicologia do Esporte, atuação em Psicologia do Esporte, intervenção em Psicologia do Esporte, foram utilizados nas buscas. As buscas ocorreram entre janeiro e março de 2018.

Diante do exposto, o presente artigo apresenta um corte temporal, que nos levou a investir sobre o assunto, em especial no Brasil. Nessa perspectiva, optou-se por apresentar estudos que fazem parte do estado da arte da Psicologia do Esporte, utilizando os que se encontrassem publicados entre 1980 e 2017. Então, delimitamos o tempo e o foco temático, mas não a pesquisa em si, que é rica em detalhes e conteúdo. O período escolhido foi proposital, pois se trata do intervalo de tempo em que a Psicologia do Esporte inicia-se enquanto área de atuação nos clubes e enquanto área de ensino nas universidades no Brasil, o que nos permite dialogar acerca do estado da arte.

\section{Psicologia do Esporte - estado da arte}

Os avanços da Psicologia do Esporte refletem as significativas descobertas e pesquisas pelas quais passam tanto a Psicologia como o Esporte (VIEIRA et al., 2010), de modo geral. Tais áreas se sedimentam como ricas desbravadoras no mundo da Ciência e, dessa forma, evoluem junto com as mais emergentes no espaço acadêmico-científico. Numa reflexão mais apurada, pode-se dizer que o Esporte galgou um espaço maior ao dar vazão aos estudos laboratoriais em áreas interdisciplinares, saindo do eixo "escola" e "fisiologia", conferindo mais abrangência e um olhar plural às Ciências do Esporte (SCHIAVON; MACHADO, 2012).

Por outro lado, a Psicologia Moderna também avança na direção de áreas emergentes e, ao delinear uma leitura qualitativa às suas pesquisas, possibilita que muitas das questões tratadas por outros segmentos acadêmico-científicos pudessem ser abarcados pelos seus pesquisadores, visto que a Psicologia não se limita ao ambiente clínico. Aqui entra outra questão que garante evolução em direção ao estado da arte: estudar ou pesquisar ou aplicar a Psicologia do Esporte foge do ambiente clínico convencional e, para tanto, a interdisciplinaridade aponta caminhos já definidos como satisfatórios.

Estudos publicados por Schiavon e Machado (2012) indicam que a Psicologia do Esporte tem caráter interdisciplinar, ligando-se às interfaces do esporte, o que possibilita sua alocação na Educação Física, no Esporte e na Psicologia, quando não na Sociologia ou Antropologia do Esporte. Esses autores argumentam que muito da produção científica assume esse olhar interdisciplinar, o que é corroborado por Vieira, Nascimento Jr. e Vieira (2013) que destacam a prevalência de publicações de artigos da Psicologia do Esporte em periódicos da Educação Física $(86,97 \%)$ em comparação aos periódicos de Psicologia $(13,03 \%)$.

Somado ao exposto acima, nota-se um aumento na produção científica na área de Psicologia do Esporte (AOYAGI et al., 2012; FRASCARELI, 2008; MACHADO; TERTULIANO, 2017; SCHIAVON; MACHADO, 2012; TERTULIANO, 2016; VIANA-MEIRELES et 
al., 2015; VIEIRA et al., 2010; VIEIRA; NASCIMENTO JR; VIEIRA, 2013;). No entanto, mesmo com esse aumento do volume de produção de conhecimento acerca da Psicologia do Esporte, percebe-se que os estudos tendem a se direcionar para algumas temáticas clássicas, como motivação, estresse e ansiedade (VIEIRA et al., 2010). Surge, então, uma dúvida: apenas esses conhecimentos são adequados para formação e atuação profissional?

Quando se trata dessa questão: adequação para formação e atuação, os mesmos estudiosos, que representam grandes laboratórios de estudos e pesquisas brasileiros e com grandes interferências na sociedade internacional da área, preferem direcionar a discussão sobre atuação profissional, sobre a questão de a disciplina de Psicologia do Esporte fazer parte da Psicologia ou de outras áreas, como a Educação Física. A consequência disso é uma briga territorial que se instala na sociedade mercantilista-profissional, criando um abismo que atrasa o desenvolvimento da área (SCHIAVON; MACHADO, 2012). Esse é um dado expressivo e interessante, visto que a Psicologia do Esporte nasceu interdisciplinar, mas tentam torná-la intradisciplinar, com olhares individualistas de classe (RUBIO, 2002; SCHIAVON; MACHADO, 2012).

Essa demanda leva os especuladores a direcionarem seus focos para a atuação, pautando-se em leis e regimentos para demonstrar sua importância para sociedade. Exemplo disso é a resolução 014/00 de 2000: de acordo com o Conselho Federal de Psicologia, a Psicologia do Esporte é uma especialidade da Psicologia, regulamentando o exercício profissional e a formação dos psicólogos (RUBIO, 2003), incluindo-a como área da formação nos cursos de Graduação em Psicologia. Diferente daquilo que acontece nos EUA e na Europa, conforme comprovaram Machado e Brandão (2008).

Ainda hoje, os cursos de Psicologia, em sua maioria, não oferecem a disciplina de Psicologia do Esporte na grade, bem como há pouca oferta da mesma nos cursos de especialização, dentro de suas faculdades. Somando-se a isso, os cursos que a ofertam focam em treinamento psicológico, controle autógeno, dinâmicas de grupo, autofala etc. (FRASCARELI, 2008). Nessa concepção, onde fica o Esporte e o Atleta? As aproximações são vagas e remotas, demandando uma maior aproximação ao espectro interdisciplinar da área, como visto no restante do mundo (LAVOURA; MELO; MACHADO, 2007).

\footnotetext{
A Psicologia do Esporte busca tratar de pessoas inseridas no esporte, sejam elas os atletas, os dirigentes, seus familiares ou agregados, árbitros e demais integrantes deste macro contexto, exercendo um fluxo de conhecimentos, inter-relacionando os conhecimentos advindos das diferentes ciências e não assumindo apenas uma área. Nesta visão, a Psicologia do Esporte aceita uma visão de homem e as questões de como cuidar dos problemas deste homem, além de se preocupar com onde ele se encontra e como suas características psicológicas podem influenciar o rendimento (MACHADO, 2008a, p.102).
}

Assim, numa ampla visão do macrossistema real, a Psicologia do Esporte está estreitamente relacionada às Ciências do Esporte, associando aspectos biológicos e pragmáticos e a intervenção direta no desempenho esportivo. Uma visão plural e polifônica é ideal para o entendimento de um movimento igualmente plural e polifônico, conforme sugerem os constructos teóricos da teoria bioecológica, atual inovadora vertente de estudos e pesquisas na área da Psicologia do Esporte, tendo como seu representante máximo Urie Bronfenbrenner (1979) e, como articuladores no Brasil, os pesquisadores Lenamar Vieira, Maria Regina Brandão e Afonso Antonio Machado, docentes universitários e membros da Sociedade Internacional de Psicologia do Esporte (ISSP).

Entende-se que faz falta um olhar mais direcionado à adequação dos conhecimentos da Psicologia e das Ciências do Esporte para atuação do profissional, seja ele psicólogo, técnico esportivo, preparador físico, fisioterapeuta, médico etc. Discutir campo de atuação, sem discu- 
tir adequação do conteúdo, é pensar pequeno, olhar unidirecionalmente e não se preocupar com o mais importante, o ser humano que está atuando como Atleta (VIEIRA et al., 2010). Essa falta de um olhar interdisciplinar é tamanha que alguns autores preferem defender a atuação de sua classe a enxergar a questão tal como ela se apresenta. Com essa visão, antes de haver debates sobre atuação, deve-se debater sobre as adequações e inter-relações dos conhecimentos advindos da Psicologia e das Ciências do Esporte, para que os mesmos sejam postos na prática, independente da formação do profissional que esteja atuando na Psicologia do Esporte (VIEIRA; NASCIMENTO JR; VIEIRA, 2013).

O debate sobre a adequação e formação se faz necessário, pois o "objeto" de trabalho, de análise, de intervenção é o ser humano, que tem seu foco de preocupação com a atuação motora e os problemas advindos dela, como seus fracassos, suas conquistas, suas metas. Nessa conduta, o profissional que queira intervir junto ao atleta deve ter, para si, que intervenção é algo além da ação profissional, é um ato de consciência, em que se exerce a intervenção e se sofre a intervenção (SCHIAVON; MACHADO, 2012, p.36).

De acordo com Frascareli (2008), as técnicas de intervenção, apesar de se mostrarem proveitosas nos estudos experimentais e nas teorizações, não se mostram da mesma forma na atuação profissional. A autora aponta que esse afastamento se dá pelo fato da Psicologia do Esporte tratar de um atleta ideal, pronto para ser classificado e treinado, e não do atleta real que deve ser apoiado, cuidado e/ou ouvido. Geralmente a proposta tem sido clínica, o que não se enquadra nas adequações emergenciais sugeridas por estudiosos clássicos da área (BRANDÃO; MACHADO, 2008; MACHADO, 2006).

Os estudos clássicos nacionais quando realizados por profissionais sem conhecimento específico da área de atuação, em sua maioria, determinam padrões de intervenção que não respeitam a bagagem cultural, social, intelectual, moral, religiosa e biológica de cada atleta (MACHADO, 2008b). Esse processo dificulta abordagens que sintonizam o momento esportivo como uma atuação do homem contemporâneo no não-lugar, no não-tempo, conforme percebemos em jogos mundiais, olímpicos e pan-americanos. De acordo com Calvo et al. (2010), a inserção do psicólogo no meio esportivo amplia os horizontes da Psicologia enquanto ciência e profissão, com consequências diretas na pesquisa e na atuação de profissionais em um campo em desenvolvimento. Entretanto, esse olhar apenas de classe precisa ser revisto, pois as Ciências do Esporte têm também uma contribuição tão importante quanto a Psicologia para o rendimento esportivo.

Além disso, limitar-se a atuação em função apenas de uma formação básica (graduação) remete a uma grande problemática da área que diz respeito à terminologia adotada. Muitos assumem a denominação Psicologia do Esporte e outros argumentam que deva ser Psicologia no Esporte, já que o atleta, muitas vezes, precisa de tratamento clínico. Botelho (2015) sugere a aproximação de um aconselhamento psicológico sem direcionamento de fundamentação teórica, portanto não-clínica. Assim, espera-se que se rompa a barreira do "inovismo" quando se trata de Psicologia do Esporte. Uma vez que a "clínica" de atuação do psicólogo do esporte passa ser no ambiente de trabalho do atleta: quadra, campo, piscina, pista, sala de dança, ambiente esportivo, ou seja, no espaço em que se desenvolve a atividade esportiva. Busca-se assim uma adequação de ambiente ao contexto em que o atleta vive, envolvendo técnico, companheiros de equipe, torcida, arbitragem, dentro da abordagem bioecológica (BRONFENBRENNER; MORRIS, 1998).

Quando se fala da atuação do profissional da Psicologia do Esporte entende-se que não será possível termos um profissional que seja "expert" numa modalidade ou atividade física apenas. O profissional ideal precisa estar preparado para uma abordagem que atenda aos universos conjuntos. Deve ser sempre contextualizado e atualizado, de modo a ter conhecimento 
das iniciações esportivas, dos ciclos de treinamentos e das jornadas competitivas: esse é seu contexto de trabalho e esse perfil dará a conotação de dinamismo e arrojo diante de suas funções. Numa visão mais micro, pensando na atuação em várias frentes de trabalho, baseado em Weinberg e Gould (2017, p. 29), tem-se um determinado endereçamento que facilita a compreensão das funções presentes na contemporaneidade:

1. Pesquisador - desenvolvendo o conhecimento, através de estudos e pesquisas; 2. Professor - desenvolvendo/ transmitindo o conhecimento obtido pelas pesquisas; 3 . Especialista em diagnóstico psíquico - avaliando e interpretando o potencial atlético (psicólogo, exclusivamente); 4. Otimizador - atuando junto aos envolvidos nos programas de atividades físicas, favorecendo o engajamento e aderência do grupo naquela atividade; 5. Consultor - para a condução do programa, na resolução de uma situação conflituosa.

No que diz respeito à atuação, ainda existem perspectivas diferentes das ditadas pelos países da América do Norte e da Europa. O modelo brasileiro não atende a nenhum desses perfis e essa falta de enquadramento dificulta uma evolução mais consistente da área. Por necessitar de uma formação acadêmica específica, ela fica na dependência do desenvolvimento profissional e científico da Psicologia, das Ciências do Esporte (área do conhecimento interdisciplinar que está ligada ao estudo das técnicas, métodos de treinamento e estratégias de preparação física e psíquica de pessoas e equipes competitivas) e do profissional que atuará utilizando-a como ferramenta.

A interdisciplinaridade leva ao conhecimento de disciplinas e profissões nas quais os comportamentos motores e, não necessariamente, os esportes passam a ser os aspectos principais de investigação (WEINBERG; GOULD, 2017). Além de que as pessoas a serem trabalhadas por essa ciência são praticantes dos mais variados níveis de destreza, modalidades e idades, ou seja, do iniciante ao experiente, da dança ao esporte olímpico, do recém-nascido ao idoso, do amputado ao fisiculturista. E toda essa clientela necessita de um acolhimento e aconselhamento à luz da Psicologia do Esporte. Diante disso, a Psicologia do Esporte pode ser compreendida como o estudo científico de pessoas e seus comportamentos em atividades de cunho esportivo, bem como a aplicação do conhecimento para intervenção (WEINBERG; GOULD, 2017). Dentro desse prisma, os grandes objetivos da área dizem respeito a entender como os fatores psicológicos afetam o desempenho físico e como a participação em esportes afeta o desenvolvimento psicológico, a saúde e o bem-estar de uma pessoa.

Olhar para a Psicologia do Esporte, em 2017, vai além do que apenas mapear fundamentações e projetos de pesquisas: significa compreender o mundo onde ela está inserida e atuante. Assim, vale reportar que em 2017 houve um Congresso Mundial de Psicologia do Esporte, em Sevilha, com mais de 4800 profissionais da área inscritos. O evento apresentou uma produção de mais de seis mil trabalhos divulgados em painéis e mesas redondas e conferências, sendo, seguramente, o maior encontro acadêmico-científico e profissional da área. A representação brasileira levou muito daquilo que aqui se produz, em especial nos laboratórios ligados aos programas de pós-graduação, que se encontram ancorados nos institutos ou cursos de Educação Física. Foram trabalhos originados de mestrados, doutorados e/ou livresdocências, produzidos numa escala vertiginosa em especial no eixo São Paulo-Paraná, com ramificações em Brasília, Santa Catarina, Minas Gerais, Rio Grande do Sul e uma representatividade ainda acanhada, mas séria e crescente de Pernambuco, Ceará e Mato Grosso.

Pensar no estado da arte da Psicologia do Esporte brasileira significa pensar nas dificuldades locais de estudiosos que se empenham em seus aprofundamentos e direcionamentos profissionais sem muito apoio de entidades de fomento e, muitas vezes, em luta contra seus órgãos de classe que não percebem a interdisciplinaridade com muita clareza e adequação. Com isso, os estudos sobre lesões psicológicas e medo e vergonha no Esporte, que 
se seguem, são fiéis representantes do que se apresentou no Congresso Mundial e merecem destaque neste artigo, configurando uma das preocupações reais daqueles que se enveredam pelas trilhas da Psicologia do Esporte no Brasil.

\section{Considerações finais}

Enquanto área observa-se que a Psicologia do Esporte é considerada interdisciplinar, pois se utiliza de conhecimentos oriundos da Psicologia, das Ciências do Esporte, da Sociologia, entre outras, com o intuito de compreender o fenômeno psicológico envolvido entre as particularidades de cada pessoa e a prática do Esporte. Talvez seja essa pluralidade de conhecimentos e procedimentos que fazem da Psicologia do Esporte uma área tão envolvente e sempre emergente. Ela se adapta e avança com a força da sociedade em que está inserida, trazendo à tona princípios básicos da sociologia, da psicologia, da antropologia, da filosofia, do esporte e demais ciências, garantindo sua evolução e sedimentação à medida que essas áreas passam a dialogar com mais frequência e consistência.

Assim, entender a Psicologia do Esporte como área de apenas uma formação (graduação) é um tanto reducionista e remete ao territorialismo dos órgãos de classe e não à verdadeira necessidade da área. Deve-se ter ciência da interdisciplinaridade que a Psicologia do Esporte alcança, dando espaços para que Profissionais de Educação Física, Fisioterapeutas, Médicos, Psicólogos, Administradores e outros, possam fazer uso dos conhecimentos oriundos das pesquisas já existentes e, mais do que isso, também façam novas pesquisas nessa área. Claro que a intervenção frente ao atleta é algo maior que visa, muitas vezes, a mudança de comportamento. Para isso a legislação estabelece que a intervenção seja feita por uma pessoa habilitada que, nesse caso, dá-se por meio do psicólogo. Ou seja, em se tratando de casos que necessitem de uma intervenção e não apenas da busca de conhecimento e do estudo em si dos fenômenos que rodeiam a Psicologia do Esporte. Assim, faz-se necessário entender que o estudo e a pesquisa são e devem continuar a ser interdisciplinares, mas que a intervenção é uma prerrogativa do psicólogo, como os textos apresentados demonstram. Contudo, deve-se reforçar que o conhecimento apenas dos funcionamentos psicológicos não é suficiente para se realizar uma boa intervenção, portanto, o profissional, além da Psicologia, deve ter também um bom conhecimento das Ciências dos Esportes.

Quanto à intervenção, espera-se que seja conduzida por profissionais qualificados e experientes para que o trabalho encontre sua adequada orientação. Tais achados permitem dizer que a vivência no ambiente esportivo e a adequada formação auxiliam o profissional que atuará na intervenção. Assim, o nível de formação universitária, somado aos aspectos culturais e ao envolvimento com o contexto esportivo, favorece a intervenção com grandes perspectivas de trabalho junto aos atletas. Todavia, o presente estudo apresenta a limitação de ser um ensaio, ou seja, o estudo da atuação na Psicologia do Esporte não se esgota por aqui. Aprofundamentos são necessários, através de novos estudos que façam análises no âmbito da intervenção, da pesquisa, do ensino, acompanhando a evolução da área.

\section{Referências}

AOYAGI, M. W.; PORTENGA, S. T.; POCZWARDOWSKI, A.; COHEN, A. B.; STATLER, T. Reflections and directions: The profession of sport psychology past, present, and future. Professional Psychology: Research and Practice, Washington, v. 43, n. 1, p. 32$38,2012$.

BARRETO, J. A. Psicologia do esporte para o atleta de alto rendimento. 1. ed. Rio de Ja- 
neiro: Shape Editora, 2003.

BOTELHO, L. R. Lesões psicológicas no esporte: principais focos de estudos. 2015. 61f. Dissertação (Mestrado em Desenvolvimento Humano e Tecnologias) - Instituto de Biociências, Universidade Estadual Paulista, Rio Claro, 2015.

BRANDÃO, M. R. F.; MACHADO, A. A. Coleção psicologia do esporte e do exercício: Aspectos Psicológicos do Rendimento Esportivo. São Paulo: Atheneu, 2008.

BRONFENBRENNER, U. The ecology of human development: Experiments by nature and design. Cambridge: Harvard University Press, 1979.

BRONFENBRENNER, U.; MORRIS, P. A. The ecology of developmental processes. In: DAMON, W.; LERNER, R. M. (Ed.). Handbook of child psychology: Vol. 1. Theoretical models of human development. New York: John Wiley, 1998. p. 993-1028.

CALVO, T. G. G.; CERVELLÓ, E.; JIMÉNEZ, R.; IGLESIAS, D.; MURCIA, J. A. M. A. M. Using self-determination theory to explain sport persistence and dropout in adolescent athletes. The Spanish Journal of Psychology, Madrid, v. 13, n. 2, p. 677-684, nov. 2010.

CAMPBELL, D. F.; MACHADO, A. A. Ensuring quality in qualitative inquiry: Using key concepts as guidelines. Motriz, Rio Claro, v. 19, n. 3, p. 572-579, 2013.

FRASCARELI, L. S. Interfaces entre Psicologia e Esporte: sobre o sentido de ser atleta. 2008. 200 f. Dissertação (Mestrado em Psicologia) - Instituto de Psicologia, Universidade de São Paulo, São Paulo, 2008.

GILL, D. L. Psychological dynamics of Sport. Champaign: Human Kinetics, 1986.

LAKATOS, E. M.; MARCONI, M. A. Metodologia científica. 6. ed. São Paulo: Atlas, 2011.

LAVOURA, T. N.; MELO, C. C.; MACHADO, A. A. Estados emocionais na Prática esportiva: Relações entre medo e vergonha no Contexto. Revista Brasileira Ciência e Movimento, Brasília, v. 15, n. 19, p. 79-88, 2007.

MACHADO, A. A. Psicologia do esporte: da educação física escolar ao esporte de alto nível. Rio de Janeiro: Guanabara Koogan, 2006.

. Liderança: novas perspectivas no futebol. In: BRANDÃO, M. R. F.; MACHADO, A. A.; MEDINA, J. P.; SCAGLIA, A. J. (Ed.). Futebol, psicologia e produção do conhecimento. 3. ed. São Paulo: Atheneu, 2008a. p. 95-112.

Mídia e esporte: canais de interferências psicológicas. In: BRANDÃO, M. R. F.; MACHADO, A. A. (Ed.). Aspectos psicológicos do rendimento esportivo. 2. ed. São Paulo: Atheneu, 2008b. p. 12-32.

Psicologia do esporte, Desenvolvimento humano e Tecnologias: o que e como estudar. 1. ed. Várzea Paulista: Editora Fontoura, 2014.

MACHADO, A. A.; TERTULIANO, I. W. Educação Física e Esportes: novos caminhos. 1. 
ed. São Paulo: Alexa Cultural, 2017.

RUBIO, K. Origens e evoluções da Psicologia do Esporte no Brasil. Revista Bibliográfica de Geografia Y Ciencias, Barcelona, v. VII, n. 373, p. 1-8, 2002.

Psicologia do Esporte: teoria e prática. São Paulo: Casa do Psicólogo, 2003.

SCHIAVON, M. K.; MACHADO, A. A. Psicologia do Esporte e a formação do profissional de Educação Física. Encontro: Revista de Psicologia, Londrina, v. 15, n. 22, p. 37-51, 2012.

TERTULIANO, I. W. Processo de expatriação de voleibolistas: Concepções Bioecológicas. 2016. 300 f. Tese (Doutorado em Desenvolvimento Humano e Tecnologias) - Instituto de Biociências, Universidade Estadual Paulista, Rio Claro, 2016.

VIANA-MEIRELES, L. G.; BOMFIM, Z. A. C.; CONDE, E. F. Q.; OLIVA, A. D. Reflexões sobre o trabalho do psicólogo do esporte em uma academia de ginástica. Motrivivência, Florianópolis, v. 27, n. 46, p. 171-184, 2015.

VIEIRA, L. F.; NASCIMENTO JR, J. R. A.; VIEIRA, J. L. L. O estado da arte da pesquisa em Psicologia do Esporte no Brasil. Revista de Psicología del Deporte, Barcelona, v. 22, n. 2, p. 501-507, 2013.

VIEIRA, L. F.; VISSOCI, J. R. N.; OLIVEIRA, L. P.; VIEIRA, J. L. L. Psicologia do esporte: Uma área emergente da psicologia. Psicologia em Estudo, Maringá, v. 15, n. 2, p. 391-399, 2010.

WEINBERG, R. S.; GOULD, D. Fundamentos da psicologia do esporte e do exercício. 6. ed. Porto Alegre: ArtMed, 2017.

Recebido em: 06/06/2018

Revisado em: 31/08/2018

Aprovado em: 11/12/2018

Endereço para correspondência:

ivanwallan@gmail.com

Ivan Wallan Tertuliano

Departamento de Educação Física

Centro Universitário Adventista de São Paulo, UNASP - Campus São Paulo.

Estrada de Itapecerica, 5859

Capão Redondo

05858001 - São Paulo, SP - Brasil 